\title{
Translucency of Human Dental Enamel
}

\author{
R.H.W. BRODBELT, W.J. O'BRIEN, P.L. FAN, J.G. FRAZER-DIB, and R. YU \\ Surface Science Laboratory, Dental Research Institute, University of Michigan, Ann Arbor, Michigan \\ 48109
}

Translucency of human dental enamel was determined by total transmittance of wavelengths from 400 to $700 \mathrm{~nm}$. The transmission coefficient at 525 $n \mathrm{~m}$ was $0.481 \mathrm{~mm}^{-1}$. Total transmission of light through human dental enamel increased with increasing wavelength. Human tooth enamel is more translucent at higher wavelengths. The translucency of wet human enamel and enamel after dehydration was also measured by total transmittance. The transmission coefficient at 525 $n m$ decreased from 0.482 to $0.313 \mathrm{~mm}^{-1}$ after dehydration and was reversed on rehydration. The decrease in translucency occurred as a result of the replacement of water around the enamel prisms by air during dehydration.

J Dent Res 60(10):1749-1753, October 1981

\section{Introduction.}

The appearance of a natural tooth is a combination of its optical properties. The esthetics of a restoration depend on its resemblance to the natural tooth structure. Shade selection and color parameters of dental materials have been subjects of investigation. ${ }^{14}$ Optical properties of tooth structures are also used in oral diagnosis and caries detection. ${ }^{5-7}$ Although the optical properties of human dental enamel form the basis for esthetics matching and caries detection, only some of its optical properties have been determined. ${ }^{8}$

Translucency is the relative amount of light transmitted through a material. One method of measuring translucency is by determining total transmission, including scattering, using a spectrophotometer with an integrating sphere. Translucency of a material can be expressed as a transmission coefficient, $t_{c}$, as the relative amount of light passing through the unit thickness of the material. ${ }^{9}$ The transmission coefficients

Received for publication October 21, 1980 Accepted for publication March 15, 1981 This study was supported by research funds from Dental Research Institute Award DE-0273114 from the National Institute of Dental Research, National Institutes of Health, Bethesda, MD 20205. for dental porcelain have been reported by Brodbelt et al. ${ }^{10}$ The purpose of the first part of this investigation was to determine the transmission coefficients of wet human dental enamel at different wavelengths.

Spitzer and ten Bosch stated that between five $\min$ and $48 \mathrm{~h}$ after the human enamel samples were taken out of the water, there was no influence of water content on optical measurements of both total reflectance and transmission. ${ }^{8}$ Boorsboom, ten Bosch, and ten Cate also stated that their human enamel samples, which had been stored in water and then allowed to dry, showed a $10 \%$ increase in reflectance for the first $20 \mathrm{~min}$, thereafter showing a $1.5 \%$ increase per h. ${ }^{11}$ The authors of these two latter papers observed the influence of water content on the sample of human enamel within the first few min of drying time, but chose to make their investigations in the latter, more stable, drying periods. Therefore, they indirectly recognized the immediate unstable period of drying human enamel which occurs just after being removed from its wet environment.

Several phenomena occur that influence the optical properties in the shade-taking process, including 1) the effect of film thickness on surface reflection; 2) the effect of water on bulk translucency; and 3 ) the effect of water content below the tooth surface. It was the purpose of the second part of this study to investigate the effect of dehydration and rehydration on the translucency of human dental enamel. This would have clinical significance in determining the optimal conditions for shade matching and caries detection by transillumination.

\section{Materials and methods.}

Wet enamel. - Thirty-six disks of human dental enamel, approximately $8.5 \mathrm{~mm}$ in diameter and $1 \mathrm{~mm}$ thick, were cut from labial surfaces of extracted maxillary central incisors. The teeth were selected at random, provided they were of substantial size and without restorations, fractures, or caries. 
The labial enamel of each tooth was reduced to a circular outline of about $8.5 \mathrm{~mm}$ diameter by use of a diamond bur. The surface was polished using a metallurgical polishing wheel and 600-grit silicon carbide paper." The tooth was then mounted for sectioning by a low speed saw. $\dagger$ Care was taken not to leave dentin on the sample disks. The samples were then stored in distilled water at room temperature.

The average thickness of each sample was obtained by measuring at five points within an external diameter of $6 \mathrm{~mm}$, using a micrometer with point contacts.

A spectrophotometer with an integrating sphere assembly $\S$ was used for transmission measurements. The sample holder described by Brodbelt et al. ${ }^{10}$ was modified to allow immersion of the samples in distilled water during measurement. Three concentric brass tubes, the smallest having an internal diameter of $6.2 \mathrm{~mm}$, were used to hold the sample in place, as well as to eliminate light leakage at the edge of the sample. The rings also maintained a standard area of 30.2 $\mathrm{mm}^{2}$ for all samples during transmission measurements. The sample chamber was covered by glass slides to provide an aqueous environment for the enamel samples.

For total transmission measurements, the samples were placed at the entrance port of the integrating sphere. Barium sulfate standards were used to complete the integrating sphere (Fig. 1). The intensity of the beam passing through the samples was continuously recorded for wavelengths from 400 to $700 \mathrm{~nm}$. A transmission spectrum and digital data record were obtained for each measurement with the light beam entering the samples from the labial surface. Four measurements were made with each sample rotated $90^{\circ}$ from the previous measurement.

If $t_{c}$ is the transmission coefficient or fractional amount of incident radiation transmitted through unit thickness of material, then the optical transmission $\left(1 / \mathrm{I}_{\mathrm{o}}\right)$ through a thickness of $x$ units of material is given by the equation: ${ }^{9}$

*Wet-or-dry Tri-M-ite Paper 600 P54, 3M Company, St. Paul, MN 55101

fIsomet 11-1180 Low Speed Saw, Buehler, Ltd., Evanston, IL 60204

$\S$ Beckman Acta C-III UV-visible Spectrophotometer, Beckman Instruments, Inc., Irvine, CA 92664

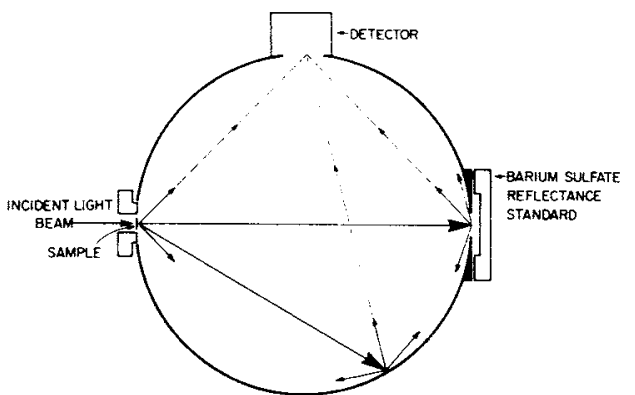

Fig. 1 - Schematic diagram for measuring translucency of dental enamel using a spectrophotometer with an integrating sphere.

$$
\mathrm{I} / \mathrm{I}_{\mathrm{o}}=\mathrm{t}_{\mathrm{c}} \mathrm{x}^{\mathrm{s}}
$$

Eq. 1

The values of the transmission coefficient were calculated for each sample at wavelengths of $425,525,625$, and $700 \mathrm{~nm}$. The $95 \%$ confidence limits for the transmission coefficients were calculated at these four wavelengths. ${ }^{12}$

Dehydrated enamel. - Six samples of human dental enamel, used for translucency determinations, were selected for this investigation. To simulate the clinical conditions of air drying teeth undergoing restorative treatment, a ten-second air drying with oilfree compressed air was used. For controlled desiccation, a desiccator with silica gel was used. Each sample was placed at an angle against a glass slide exposing all surfaces to dehydration. Three sequential desiccation periods of $15 \mathrm{~min}$ were used to simulate an average dental office treatment. Rehydration of enamel samples was done by immersion in distilled water for at least $48 \mathrm{~h}$.

Total transmission measurements were performed using the spectrophotometer with an integrating sphere which was used earlier for wet enamel. Wet enamel samples were measured with water in the sample chamber of the holder. Dehydrated enamel samples were measured with air in the sample chamber, and the transmission coefficients were calculated using Equation 1.

The relative translucency at different degrees of dehydration was calculated for each sample using the equation:

relative translucency $=\frac{t_{c}}{t_{c}(w e t)} \times 100$. Eq. 2

The relative translucency of wet enamel was taken as 100 . 


\section{Results.}

Wet enamel. - A representative transmission spectrum of human dental enamel is shown in Fig. 2. All enamel samples showed increasing transmission with increasing wavelength from 400 to $700 \mathrm{~nm}$. The mean values of transmission coefficient and confidence intervals at the four wavelengths are listed in Table 1 and are shown graphically in Fig. 3.

Representative transmission spectra of human dental enamel at different degrees of dehydration are shown in Fig. 4. The transmission coefficients and relative translucency are listed in Tables 1 and 2, respectively. Graphical presentations are shown in Fig. 5. An analysis of variance showed that there were significant differences in mean values at $p<0.05$. Multiple comparisons were made using the StudentNewman-Keuls test ${ }^{12}$ at $\alpha=0.05$. The translucency of human dental enamel was significantly altered by dehydration and was fully restored by rehydration. There was no significant difference in the translucency of dental enamel dehydrated in a desiccator for 15,30 , and $45 \mathrm{~min}$. However, these translucency values were significantly lower than those of dental enamel dehydrated by a tensecond exposure to a compressed air stream.

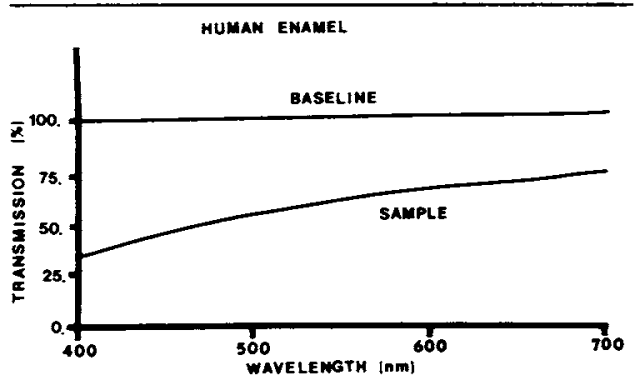

Fig. 2 - Transmission spectrum of human dental enamel.

TABLE 1

TRANSMISSION COEFFICIENTS OF WET HUMAN DENTAL ENAMEL AT VARIOUS WAVELENGTHS

\begin{tabular}{ccc}
\hline $\begin{array}{c}\text { Wavelength } \\
(\mathrm{nm})\end{array}$ & $\begin{array}{c}\text { Transmission } \\
\text { Coefficient } \\
\left(\mathrm{mm}^{-1}\right)\end{array}$ & $\begin{array}{c}95 \% \text { Confidence } \\
\text { Interval } \\
\left(\mathrm{mm}^{-1}\right)\end{array}$ \\
\hline 425 & $0.327(0.030)^{*}$ & $0.322-0.332$ \\
525 & $0.481(0.039)$ & $0.475-0.488$ \\
625 & $0.578(0.046)$ & $0.571-0.586$ \\
700 & $0.629(0.050)$ & $0.621-0.638$ \\
\hline
\end{tabular}

*Standard deviations in parentheses.

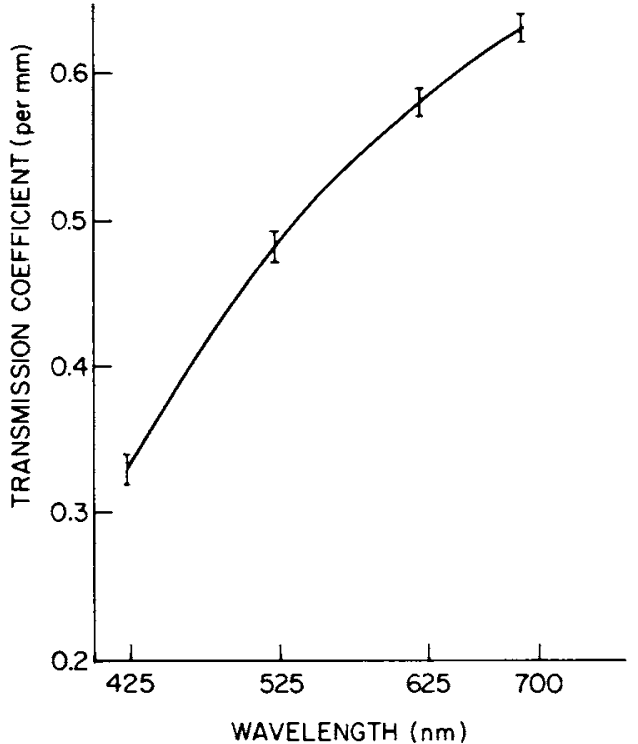

Fig. 3 - Transmission coefficient of human dental enamel at various wavelengths.

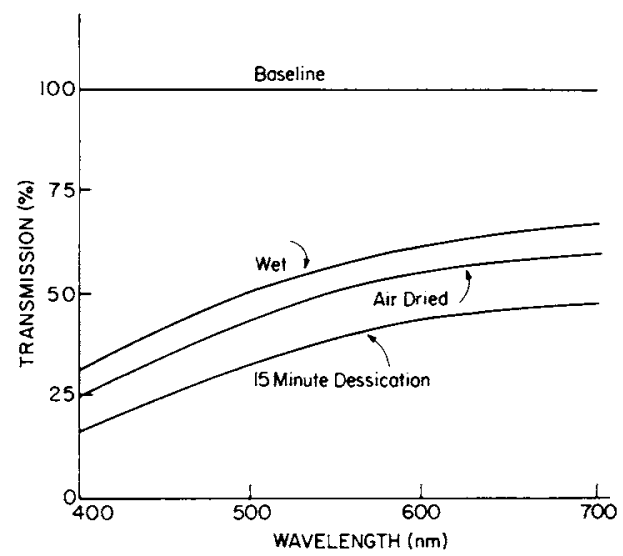

Fig. 4 - Transmission spectra of human dental enamel at different degrees of dehydration.

\section{Discussion.}

The values of the transmission coefficient of human dental enamel were different at wavelengths of $425,525,625$, and $700 \mathrm{~nm}$. The $95 \%$ confidence intervals for the transmission coefficients at these four wavelengths did not overlap, indicating significant differences among the mean values.

It was observed that there was no difference in transmission intensity when the incident beam entered a sample either from the labial direction or from the lingual direction. Furthermore, there were no significant 
TABLE 2

TRANSMISSION COEFFICIENTS $\left(t_{c}\right) M^{-1}$ OF DENTAL ENAMEL AT DIFFERENT DEGREES OF DEHYDRATION

\begin{tabular}{llccc|}
\hline \hline Enamel Condition & \multicolumn{4}{c}{ Wavelength $(\mathrm{nm})$} \\
\hline & \multicolumn{1}{c|}{425} & 525 & 625 & 700 \\
Wet & $0.329(0.033)^{*} \mid$ & $0.482(0.047)$ & $0.580(0.053)$ & $0.633(0.057)$ \\
Rehydrated $(48 \mathrm{~h})$ & $0.32(0.03)$ & $0.477(0.049)$ & $0.570(0.056)$ & $0.623(0.059)$ \\
Ten-second air blast & $0.252(0.053)$ & $0.398(0.063)$ & $0.473(0.070)$ & $0.511(0.072)$ \\
15-minute desiccation & $0.182(0.035)$ & $0.321(0.045)$ & $0.391(0.047)$ & $0.426(0.046)$ \\
30-minute desiccation & $0.179(0.032)$ & $0.316(0.043)$ & $0.385(0.043)$ & $0.419(0.041)$ \\
45-minute desiccation & $0.174(0.034)$ & $0.313(0.044)$ & $0.381(0.046)$ & $0.414(0.046)$ \\
\hline
\end{tabular}

* Standard deviations in parentheses.

Values joined by the same line are not statistically different at $\alpha=0.05$.

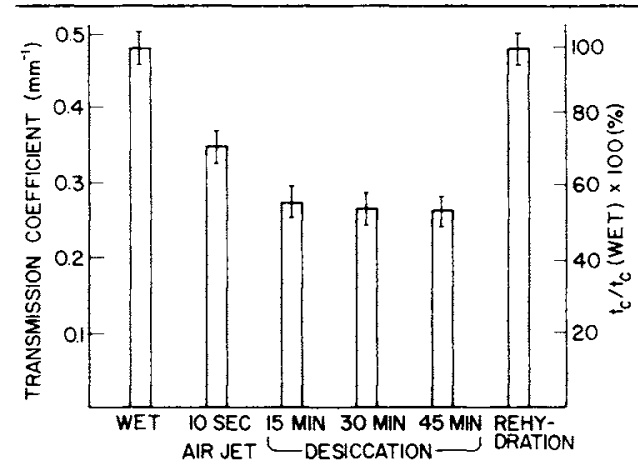

Fig. 5 - Transmission coefficients and relative translucency of human dental enamel at different degrees of dehydration.

differences in transmission intensities when the samples were rotated through the four $90^{\circ}$ orientations.

The decrease in transmission coefficient with decreasing wavelengths is most likely due to the increase in light scattering as indicated by the Rayleigh scattering equation. Similar results were reported for dental porcelains. ${ }^{10}$ The transmission coefficient value of human dental enamel at $700 \mathrm{~nm}$ is almost twice that at $425 \mathrm{~nm}$.
The transmission coefficient at $525 \mathrm{~nm}$, $0.481 \mathrm{~mm}^{-1}$, is considerably higher than the average transmission coefficient of dental porcelain enamels, $0.268 \mathrm{~mm}^{-1}$, at the same wavelengths. This indicates that the dental porcelain enamels are more opaque and may explain the observation that porcelains lack a natural appearance.

The translucency of human dental enamel decreased on dehydration as indicated by the lower transmission coefficient values and the corresponding relative translucency values. Enamel dehydrated in a desiccator reached a steady translucency value after $15 \mathrm{~min}$, since there was no statistically significant difference between the relative translucency values after $15 \mathrm{~min}$ and after $45 \mathrm{~min}$ of desiccation. At a wavelength of $525 \mathrm{~nm}$, the relative translucency of enamel stored in a desiccator was $67 \%$ lower than that of wet enamel.

Dehydration of enamel by a ten-second exposure to an air stream was used to simulate clinical treatment. The relative translucency decreased to $82 \%$ of that of wet enamel. The use of rubber dams and long operating procedures would cause even more severe dehydration and a greater decrease in translucency.

TABLE 3

RELATIVE TRANSLUCENCY $\left(t_{c} / t_{c}\right.$ WET $) X 100 \%$ OF DENTAL ENAMEL AT DIFFERENT DEGREES OF DEHYDRATION

\begin{tabular}{lcccc}
\hline \hline Enamel Condition & & \multicolumn{2}{c}{ Wavelength (nm) } \\
Wet & 425 & 525 & 625 & 700 \\
Rehydrated $(48 \mathrm{~h})$ & $100.0(0.0)^{*} \mid$ & $100.0(0.0) \mid$ & $100.0(0.0) \mid$ & $100.0(0.0) \mid$ \\
Ten-second air blast & $99.3(4.4)$ & $99.9(3.9) \mid$ & $99.7(2.4) \mid$ & $99.8(2.0) \mid$ \\
15-minute desiccation & $76.2(11.5)$ & $82.3(8.3)$ & $81.5(7.4)$ & $80.6(8.0)$ \\
30-minute desiccation & $55.0(7.0)$ & $66.7(5.9)$ & $67.1(4.8) \mid$ & $67.2(4.1) \mid$ \\
45-minute desiccation & $55.8(6.3)$ & $66.5(4.9)$ & $67.3(3.8)$ & $67.2(3.8) \mid$ \\
\hline
\end{tabular}

*Standard deviations in parentheses. Values joined by the same line are not statistically different at $\alpha=0.05$. 
Enamel in the wet state and in different stages of dehydration showed higher transmission coefficient values at higher wavelengths. This is in accordance with the Rayleigh scattering equation. Similar results on dental porcelain were reported by Brodbelt $e t$ al. ${ }^{10}$ The relative translucency values were similar at wavelengths of 525,625 , and $700 \mathrm{~nm}$, but were lower at $425 \mathrm{~nm}$. This was probably a result of increased scattering at lower wavelengths.

The translucency of dental enamel was shown to be influenced by its degree of dehydration. This could be the result of the replacement of the water around the enamel prisms by air. The effect of scattering in a heterogeneous system, such as enamel prisms surrounded by a fluid medium (e.g., water), is a function of the difference in refractive indices of the two components. The refractive index of dental enamel ${ }^{13}$ is approximately 1.7. Since the refractive index of refraction of water is 1.33 and that of air is 1.00 , a larger difference and greater scattering are produced at an enamel-air interface. Therefore, dehydrated enamel would show a lower translucency as found.

\section{Conclusions.}

Translucency of dental enamel can be expressed as a transmission coefficient, $t_{c}$, for total (direct and diffused) transmission. The values of $t_{c}$ are dependent on the wavelength of the incident light. The transmission coefficients for dental enamel are higher at longer wavelengths of the visible spectrum. Dehydration resulted in a reduction of translucency values. This decrease in translucency is explained as a result of an increased difference in refractive indices between the enamel prisms and the surrounding medium when water is replaced by air.

\section{REFERENCES}

1. PRESTON, J.D.: Color in Fixed Prosthodontics, Monograph, Los Angeles, CA: V. A. Wadsworth Hospital, 1974, 18 p.

2. CULPEPPER, W.D.: A Comparative Study of Shade Matching Procedures, $J$ Prosthet Dent 24:166-174, 1970.

3. GRAJOWER, R.; REVAN, A.; and SORIN, S.: Reflectance Spectra of Natural and Acrylic Resin Teeth, $J$ Prosthet Dent 36: 570-579, 1976.

4. DENNISON, J.B.; POWERS, J.M.; and KORAN, A.: Color of Dental Restorative Resins, J Dent Res 57:557-562, 1978.

5. DOMINKOVIC, T.: Total Reflectance in Tooth Substance and Diagnosis of Cracks in Teeth - A Clinical Study, Swed Dent J 1: 163-172, 1977.

6. WRIGHT, G.Z. and SIMON, I.: An Evaluation of Transillumination for Caries Detection in Primary Molars, J Dent Child 39:199. 202, 1972.

7. BARENIE, J.; LESKE, G.; and RIPA, L.W.: The Use of Fiber Optic Transillumination for Detection of Proximal Caries, Oral Surg 36:891-897, 1973.

8. SPITZER, D. and ten BOSCH, J.J.: The Absorption and Scattering of Light in Bovine and Human Enamel, Calcif Tiss Res 17:129$137,1975$.

9. MORGAN, J.: Introduction to Geometric and Physical Optics, New York: McGraw-Hill Book Company, 1953.

10. BRODBELT, R.H.W.; O'BRIEN, W.J.; and FAN, P.L.: Translucency of Dental Porcelains, J Dent Res 59:70-75, 1980.

11. ten BOSCH, J.J.; BOORSBOOM, P.C.F.; and ten CATE, J.M.: A Nondestructive Optical Method to Study De- and Re-mineralization of Enamel, $J$ Dent Res Spec Iss B:1027, 1979.

12. SOKAL, R.R. and ROHLF, F.J.: Biometry, San Francisco, CA: W. H. Freeman and Co., 1969, p. 139.

13. HOUWINK, B.: The Index of Refraction of Dental Enamel Apatite, Br Dent $J$ 137:472$475,1974$. 